\title{
Varying Characteristics of Bipolar Transistors with Emitter Contact Window Width
}

\author{
J. Fu, S. Mijalkovic, W. J. Eysenga, H. W. van Zeijl and W. Crans \\ Delft Institute of Microelectronics and Submicron Technology (DIMES) \\ Feldmannweg 17, P. O. Box 5053, 2600 GB, Delft, The Netherlands \\ E-mail: fujun@ectmuxs1.et.tudelft.nl,w.crans@its.tudelft.nl
}

\begin{abstract}
On the basis of careful selection and calibration of model parameters we performed process and subsequent device simulation on WEB bipolar transistors. As a result, we concluded, that the mechanical stresses may be responsible for the measured varying device DC characteristics with emitter contact window width.
\end{abstract}

\section{Introduction}

Fully-implanted washed-emitter-base (WEB) NPN bipolar transistors were optimized for operation in the $10-30 \mathrm{GHz}$ range with low-stress silicon nitride film as surface isolation layer [6] (Nanver et al. 1996). The DC characteristics of the transistors were observed to vary with the emitter contact window width in an unexpected way. We investigated such a phenomenon with the aid of process and device simulators, i.e., TSUPERM-4 and MEDICI, respectively, based on careful selection and calibration of the corresponding model parameters. As a result, the observed variations in device characteristics with the emitter contact window width may reasonably be ascribed to the influence of the mechanical stresses induced by the silicon nitride surface isolation layer.

\section{Processing sequence}

The main steps of WEB bipolar process used for manufacturing the transistors can be described as follows. After the formation of $\mathrm{an}^{+}$arsenic buried layer on top of (100) p type Si substrate $(2-5 \Omega \mathrm{cm})$, a $0.9 \mu \mathrm{m}$ epitaxial layer doped with $1 \times 10^{16} \mathrm{~cm}^{-3}$ arsenic was grown, followed by thermal growth of $30 \mathrm{~nm}$ silicon oxide and the subsequent implantation of $\mathrm{p}^{+}$junction isolation and $\mathrm{n}^{+}$collector plug. Next low ohmic extrinsic base and link base were formed by boron implantation with doses of $3 \times 10^{15} \mathrm{~cm}^{-2}$ and $5 \times 10^{13} \mathrm{~cm}^{-2}$, respectively, both at an energy of $15 \mathrm{keV}$. Low stress silicon nitride $\mathrm{SiN}_{\mathrm{x}}$ layer with a thickness of $300 \mathrm{~nm}$ was successively deposited. All contact windows were opened through plasma etching of $\mathrm{SiN}_{\mathrm{x}}$ and pad oxide in one masking step, followed by emitter (arsenic, $40 \mathrm{keV}, 7.5 \times 10^{15} \mathrm{~cm}^{-2}$ ) and intrinsic base (boron, $20 \mathrm{keV}$, $1.75 \times 10^{14} \mathrm{~cm}^{-2}$ ) implants. All dopants were activated by a single thermal anneal for $30 \mathrm{~min}$ in an argon ambient at $950^{\circ} \mathrm{C}$. The cross-sectional view of the final device structure is shown in Fig. 1.

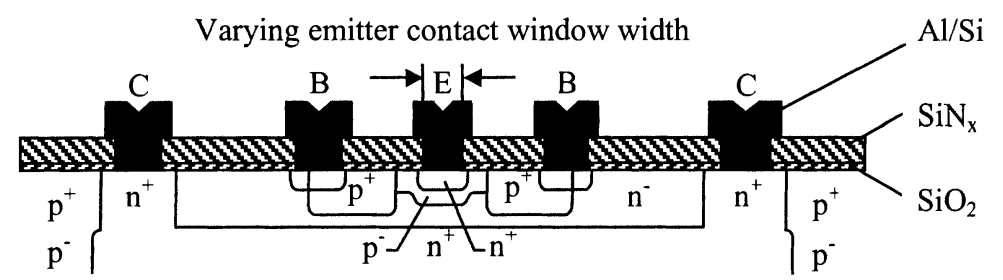

Fig. 1. Schematic cross section of WEB bipolar transistor 


\section{Simulation}

The WEB bipolar process and DC characteristics of the fabricated transistors were simulated by using TSUPREM-4 (version 6.6) and MEDICI (version 4.1), respectively. In order to improve the accuracy of process simulation, the fully coupled point defect model was selected to predict post-implant dopant diffusions during some of the critical process steps and the corresponding model parameters (e.g., dopant diffusivity, equilibrium concentration and diffusivity of silicon interstitials, and so on, see reference [2] (Fu et al. 2001)) were calibrated by using Inverse modelling driver PROFILE. It is worth noting that such a calibration procedure was based on comparisons of simulated dopant profiles given by TSUPREM-4 with the corresponding SIMS data detected from the accompanying wafers of the WEB bipolar process published in reference [6] (Nanver et al. 1996). On the other hand, Phillips unified mobility model and field dependent mobility model, which are thought to be suitable for bipolar transistor simulation, were chosen to perform the subsequent MEDICI device simulation. In accordance with the default values of the model parameters used in Phillips unified mobility model, the values of the parameters employed to describe the bandgap narrowing effect followed reference [3] (Klaassen et al. 1992). As for the recombination of carriers doping concentration dependent SRH model and Auger model were utilized with the corresponding model parameters given by reference [4] (Law et al. 1991).

\section{Results and discussions}

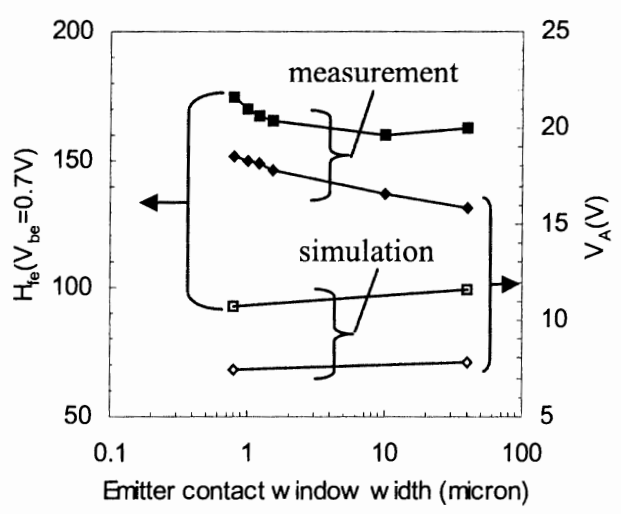

Fig. 2. Common emitter current gain $\mathrm{H}_{\mathrm{fe}}$ and Early voltage $V_{A}$ versus emitter contact window width for WEB bipolar transistors

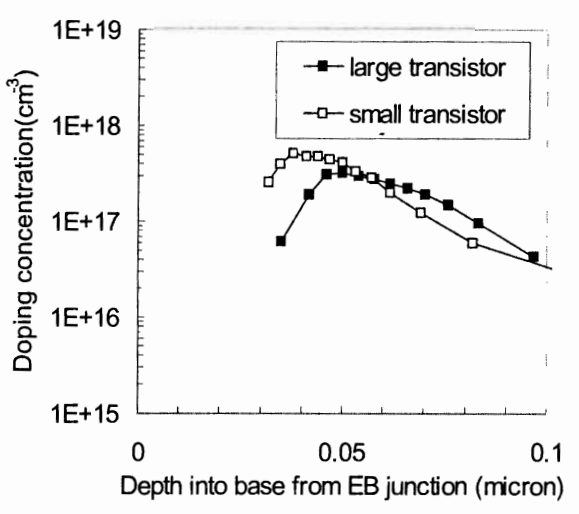

Fig. 3. Base doping profiles in the middle of emitter contact window for large and small transistors extracted by using differential capacitance method

Fig. 2 exhibits the measured common emitter current gain $\mathrm{H}_{\mathrm{fe}}\left(\mathrm{V}_{\mathrm{be}}=0.7 \mathrm{~V}\right)$ and Early voltage $\mathrm{V}_{\mathrm{A}}$ for WEB bipolar transistors as a function of emitter contact window width with a fixed emitter contact window length of $40 \mu \mathrm{m}$. For comparison the simulated $\mathrm{H}_{\mathrm{fe}}$ and $\mathrm{V}_{\mathrm{A}}$ are plotted as well in Fig. 2 for large emitter contact window width $\left(40 \times 40 \mu \mathrm{m}^{2}\right)$ and small one $\left(40 \times 0.8 \mu \mathrm{m}^{2}\right)$, respectively. It should be noted that the process conditions for fabricating the set of transistors differ somewhat in the dosages of link base and intrinsic base boron implants from those in reference [6] (Nanver et 
al. 1996) used for model parameter calibration. In fact, the nominal dosages of boron implantation used for link base and intrinsic base formation corresponding to Fig. 2 are $5 \times 10^{13} \mathrm{~cm}^{-2}$ and $1.75 \times 10^{14} \mathrm{~cm}^{-2}$, instead of $1.1 \times 10^{14} \mathrm{~cm}^{-2}$ and $2.4 \times 10^{14} \mathrm{~cm}^{-2}$ in reference [6] (Nanver et al. 1996), respectively. For this reason the boron implant dosages involved in process simulation here were scaled accordingly. It can be clearly found that the simulated $\mathrm{H}_{\mathrm{fe}}$ and $\mathrm{V}_{\mathrm{A}}$ are significant less than those from measurement for both the large device and the small device. In addition, the simulation can not predict the trend of either $\mathrm{H}_{\mathrm{fe}}$ or $\mathrm{V}_{\mathrm{A}}$ versus the emitter contact window width. For the time being we don't discuss the current gain and its complicated mechanisms. In contrast to current gain, the magnitude of Early voltage is dominantly determined by the integrated quantity of base dopants per unit area, i.e., Gummel number. Consequently the higher measured $\mathrm{V}_{\mathrm{A}}$ 's compared to the simulated ones can reasonably be explained as relatively higher Gummel numbers (averaged over the emitter contact window) in the actual devices than those in the unpatterned accompanying samples prepared for SIMS detection. Accordingly, the increase in measured $\mathrm{V}_{\mathrm{A}}$ with decreasing emitter contact window width as shown in Fig. 2 may be attributed to the increasing Gummel number with shrinkage of emitter contact window width. This assumption has been confirmed by the base doping profiles in the middle of emitter contact window extracted by means of so-called differential capacitance technique [1] (Fu et al. 2000) for the large and the small transistor, respectively, as shown in Fig. 3.

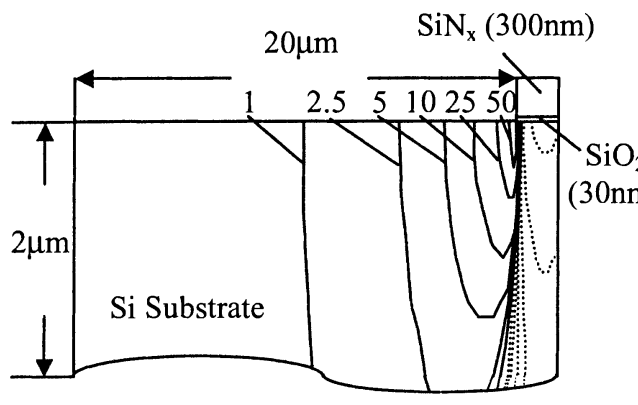

(a) $40 \times 40 \mu \mathrm{m}^{2}$

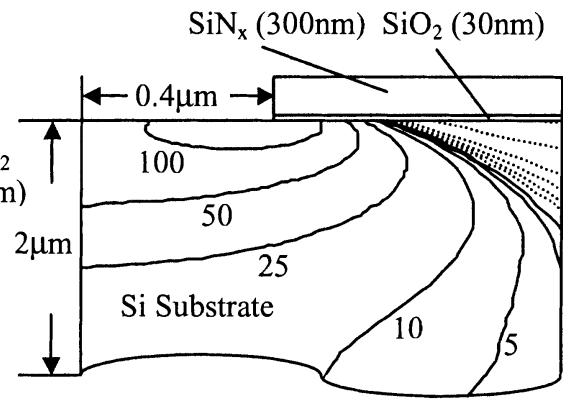

(b) $40 \times 0.8 \mu \mathrm{m}^{2}$

Fig. 4. Mechanical stress contours inside device structure for the large (a) and the small (b) transistor simulated by FLOOPS (only half of the emitter contact window is shown in consideration of symmetry of the structure)

unit: $10^{7} \mathrm{dyn} / \mathrm{cm}^{2}$; solid lines: tensile stress; dotted lines: compressive stress

A possible explanation for the emitter contact window width dependent dopant profiles is the influence of mechanical stresses induced by silicon nitride surface isolation layers on the dopant diffusions. The stress contours inside device structure for large and small transistor are obtained by FLOOPS simulation based on S. Mijalkovic's work [5] (Mijalkovic 2000) as plotted in Fig. 4 (a) and (b), respectively (only half of the emitter contact window is shown in consideration of symmetry of the structure). As we can clearly see from Fig. 4 that localized stresses are created around the edges of emitter contact windows as a result of patterning of silicon nitride layers. Moreover, the magnitude of the stresses near the center of emitter contact windows 
would be increased with decreasing window width as predicted by the FLOOPS stress analysis. It should be emphasized that we did not take any of the mechanical stress related effects into consideration during the TSUPREM-4 and MEDICI simulation. So the observed deviation of simulation from measurement regarding the DC characteristics of bipolar transistors may reasonably be ascribed to the effects of stresses. As a result, varying base doping profiles with emitter contact window width, and thus the elevated $\mathrm{V}_{\mathrm{A}}$ with decreasing emitter contact window width can be accounted for in terms of perturbation of dopant diffusions arising from influence of mechanical stresses.

\section{Conclusions}

The varying DC characteristics of WEB bipolar transistors with emitter contact window width were investigated through process and subsequent device simulation. The observed deviation of simulation from the corresponding measurement and variations in measured $\mathrm{V}_{\mathrm{A}}$ as a function of emitter contact window width led us to the conclusion that the Gummel number and hence Early voltage can be increased as a result of perturbed dopant diffusions in the presence of mechanical stresses induced by the patterned silicon nitride layer. The extracted base doping profiles inside the device structure provided further evidence in favor of our conclusion. In order to interpret varying current gain versus emitter contact window width, however, further investigations are required to gain more insight into the influence of the stress and strain states.

\section{Acknowledgement}

The authors wish to thank Dr. L. K. Nanver et al. for providing the measurement data of dopant profiles and device characteristics for WEB bipolar process. The authors also would like to thank the NWO-Technology Foundation-STW who is sponsoring this project under grant DEL. 4415.

\section{References}

[1] Fu J. , S. Mijalkovic, W. J. Eysenga, H. W. van Zeijl, and W. Crans (2000): The Influence of Mechanical Stresses on the Characteristics of Bipolar Transistors. In: Proceedings of Semiconductor Advances for Future Electronics 2000 (SAFE 2000), Veldhoven, the Netherlands

[2] Fu J. , W. J. Eysenga, and W. Crans (2001): Calibration of model parameters for process simulation on dopant ion implant and diffusion by using Inverse Modelling method. In: Proceedings of Semiconductor Advances for Future Electronics 2001 (SAFE 2001), Veldhoven, the Netherlands (to be published)

[3] Klaassen D. B. M. , J. W. Slotboom, and H. C. de Graaff (1992): Unified apparent bandgap narrowing in n- and p-type silicon. Solid-State Electronics. 35: 125-129

[4] Law M. E. , E. Solley, M. Liang, and Dorothea E. Burk (1991): Self-consistent model of minority-carrier lifetime, diffusion length, and mobility. IEEE Electron device letters. 12: 401-403

[5] Mijalkovic S. (2000): A piecewise linear Galerkin approach to stress analysis of nearly incompressible materials. Communications in Numerical Methods in Engineering. 16: 537-543

[6] Nanver, L. K. , E. J. G. Goudena, and H. W. van Zeijl (1996): Optimization of fullyimplanted NPN's for high-frequency operation. IEEE transactions on electron devices. 43: 1038-1040 